\title{
Confirmation diagnosis of Influenza A(H1N1)2009 by Belgian sentinel laboratories during the epidemic phase
}

by

\author{
Muyldermans $\mathrm{G}^{1}$, Ducoffre $\mathrm{G}^{1}$, Thomas $\mathrm{I}^{2}$, Clement $\mathrm{F}^{3}$, De Laere $\mathrm{E}^{3}$, Glupczynski $\mathrm{Y}^{3}$, \\ Hougardy $\mathrm{N}^{3}$, Lagrou $\mathrm{K}^{3}$, Léonard $\mathrm{PE}^{3}$, Meex $\mathrm{C}^{3}$, Pierard $\mathrm{D}^{3}$, Raymaekers $\mathrm{M}^{3}$, \\ Reynders $\mathrm{M}^{3}$, Stalpaert $\mathrm{M}^{3}$, Verstrepen $\mathrm{W}^{3}$, Quoilin $\mathrm{S}^{1}$
}

\section{Keywords}

Influenza, $\mathrm{A}(\mathrm{H} 1 \mathrm{~N} 1)$ pandemic, epidemiology, laboratory diagnosis

\section{Introduction}

From 13 July onwards, the Interministerial Influenza Coordination Committee announced the switch from the containment phase to a mitigation phase due to the increased number of Influenza $A(H 1 N 1) 2009$ cases $(1,2)$. This modified approach means that it is no longer necessary to conduct a virological examination for every suspicious case and that the diagnosis relied on clinical criteria again. It also required the initiation of several surveillance systems allowing the follow-up of the infection.

However, for some patients a virological confirmation was still needed due to their clinical evolution or clinical history. Because of the high amount of these requests, it was proposed to decentralise the diagnostic capacity from the Belgian National Influenza Centre (NIC) to a network of 'partner' laboratories.

Therefore, all sentinel laboratories were invited to implement the diagnostic tests, to participate in epidemiological surveillance in the frame of the sentinel laboratory network, to participate in virological surveillance by sending laboratory results or strains to be further subtyped at the NIC.

A network of 15 laboratories that responded favourably was thus installed and used as an additional tool for the virological surveillance of the Influenza $A(H 1 N 1) 2009$, allowing the identification of circulating types and subtypes.

Here we report on the data gathered by this network for the period from week 40 to week 53 of 2009.

\footnotetext{
Scientific Institute of Public Health, Operational Direction Public Health and Surveillance, Infectious Diseases in the General Population, Brussels, Belgium

2 Scientific Institute of Public Health, Operational Direction Transmitted and Infectious Diseases, Virology Diseases, Brussels, Belgium

3 Participating laboratories further listed in the acknowledgement gaetan.muyldermans@wiv-isp.be
} 


\section{Methods}

A list with all participating laboratories $(n=15)$ was published on the internet (http://www.wivisp.be/flu-surveillance/) making it available to all requestors. The analysis request form and instructions including the sample collection and transport conditions, the inclusion criteria, as well as the turnaround time for analysis were generated for each laboratory in both Dutch and French and published on the website.

Based on the inclusion criteria described in the instruction document, testing focussed on hospitalised patients and patients at risk for severe disease for whom the results of the test could influence decisions regarding care and treatment.

To support these laboratories, the analysis procedure and positive controls from positive samples were offered by the NIC. However, no restriction was given on the procedure, neither on the algorithm used.

During the study period, following data were collected: sample identifiers, patient birth date, gender and residence, and results of the antigen detection and PCR (typing A/B and subtyping $A(H 1 N 1) 2009)$ if available. Results were extracted weekly from the LIMS and collected by WIV-ISP for further data analysis.

\section{Results}

From 28 September 2009 (week 40) until 3 January 2010 (week 53), a total of 5942 samples were collected by 12 out of the 15 laboratories voluntarily participating in the network and providing their data. Different PCR procedures as well as confirmation algorithms were used by these laboratories. Four laboratories performed a screening test (antigen detection and/or Influenza A PCR) before subtyping for Influenza A(H1N1)2009 by PCR, 2 laboratories immediately performed $A(H 1 N 1) 2009$ subtyping, 5 laboratories performed the typing for Influenza $A$ and the subtyping for $A(H 1 N 1) 2009$ simultaneously, while 1 changed its algorithm during the study period.

A median of 308 samples was determined by the 12 participating laboratories, range 411596 (IQR 181-532). Two laboratories, one from the Flemish region and one from the Brussels-Capital Region, received together 3190 out of the 5942 samples (53.7\%).

Since the majority of the laboratories reporting the postal code were localised in the Flemish Region $(n=6)$ and in Brussels $(n=2)$, samples were mainly collected from patients from these regions: 3159 from Flemish region, 1403 from Brussels, 898 from the Walloon region and 482 patients with unknown residence.

Two out of the 7 laboratories performing the Influenza B typing detected Influenza B in 8 samples. As no Influenza A subtyping for seasonal H1N1 and H3N2 was performed, no information could be analysed on this matter. 
For 3139 out of the 5942 samples, an Influenza A(H1N1)2009 PCR was performed according to their confirmation algorithm. Of these, 1464 (46.7\%) samples were positive, 1649 $(52.5 \%)$ negative and $26(0.8 \%)$ indeterminate, the latter mainly due to inhibition. All further data analysis is performed on these 3139 samples.

No difference in sex ratio (M/F: 1.08) appeared with 1583 confirmed diagnoses among males and 1459 among females.

As demonstrated in figure 1 the study period included the epidemic. The peak of the epidemic was observed in week 43 when 341 cases were detected (positivity rate 65.8\%). Although a higher amount of samples were analysed in week $44(n=545)$ as compared to week $43(n=518)$, a smaller proportion of samples were found positive.

The median age of the Influenza A(H1N1)2009 positive cases was 9.8 years (IQR: 3.1-25.9). Nearly sixty-two percent $(62 \%)$ of the positive cases were found within the age group of $0-15$ years and $88 \%$ of the confirmed cases were younger than 45 years (data not shown). Based on the proportion of incidence by age group, a similar age distribution profile was observed for all participating laboratories (Figure 2). Depending on the laboratory, 75 to $99 \%$ of the confirmed cases were younger than 45 years, and $95 \%$ to $100 \%$ was younger than 65 years.

The positivity rates (number of positive results on total number of samples tested) were homogeneously distributed between 30.4 and 32.9 for all but one laboratory $(n=4)$ performing the Influenza $A$ and $A(H 1 N 1) 2009$ PCR in one run. The other laboratory reported only positive cases and therefore a positive rate could not be calculated. For the only laboratory changing from algorithm strategy during the study period, a higher positivity rate of 40.9 was notified.

Figure 1: Distribution of Influenza A(H1N1)2009 tests results, by week, between week 40 and 53, 2009, Belgium

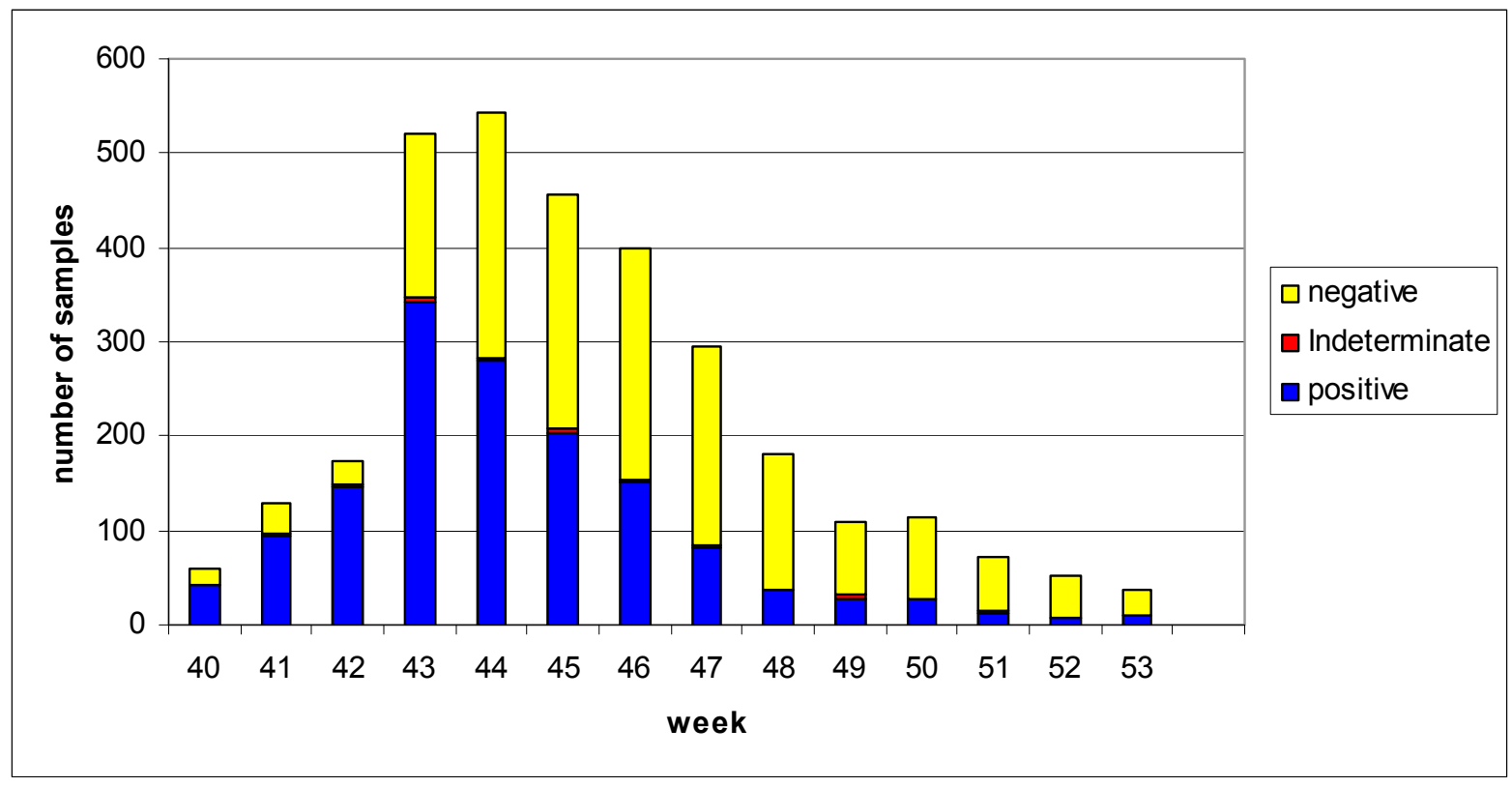


Figure 2: proportion of incidences by age group as analysed by the participating laboratories for Influenza $\mathrm{A}(\mathrm{H} 1 \mathrm{~N} 1) 2009$ cases between week 40 and 53, 2009

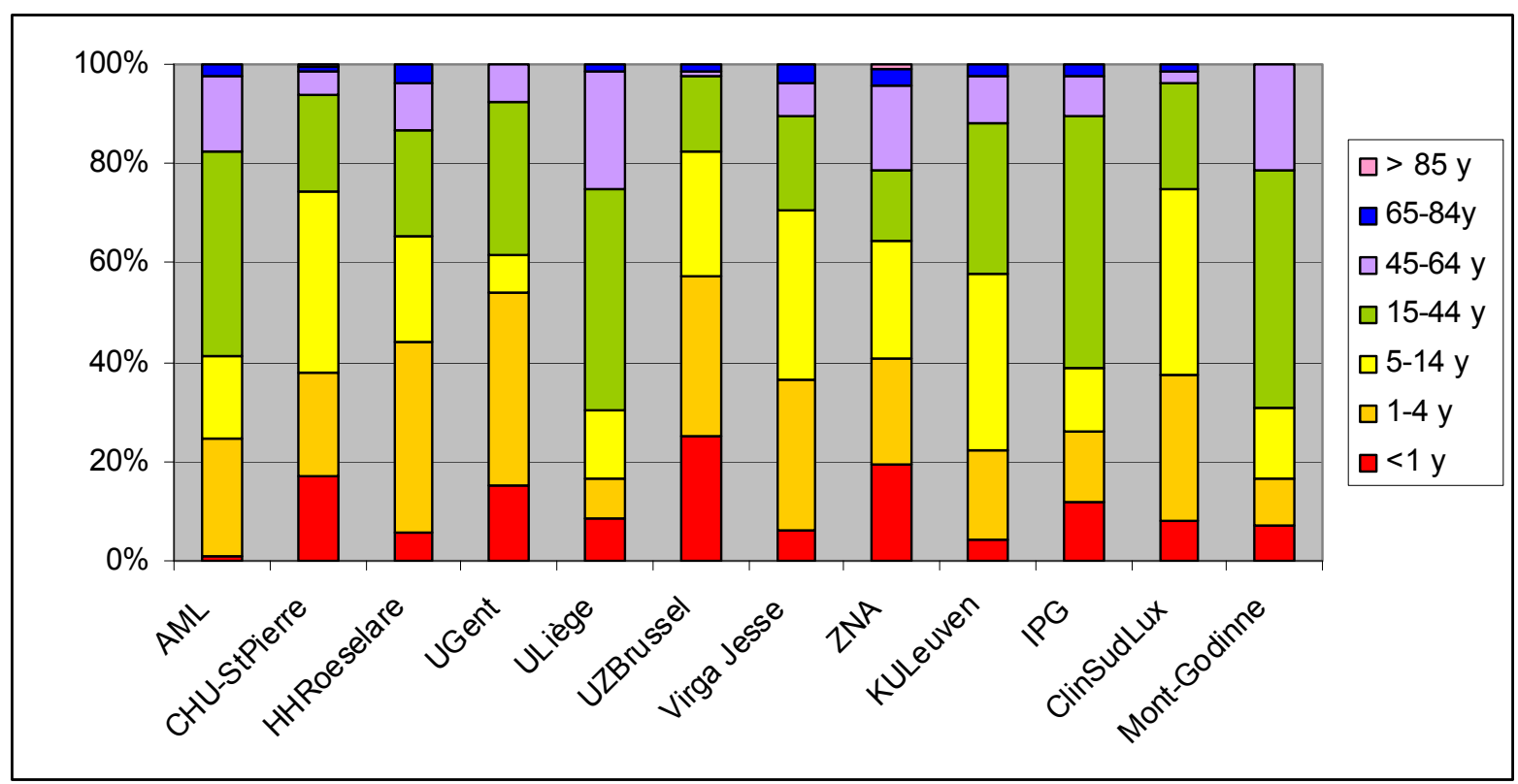

\section{Discussion}

The transfer of the Influenza virus testing to partner laboratories was promoted to increase the diagnostic capacity required to meet the increased demands. This allowed the NIC to focus its activity again on the population based surveillance, the antigenic and genetic characterisation of the strains and the anti-viral resistance monitoring.

Although the decentralisation of the activities to the participating laboratories improves the analysis turnaround time and thus the patient care, it reduces the completeness of the cohort database. The missing information for gender, residence and date of birth can be explained by the priorities defined by the individual laboratories. Therefore the decentralisation reduced the capacity and the power of the epidemiological surveillance and this information can only be provided by community-based surveillance programmes such as the SGP network $(3,4)$.

Patients included in this study were not equally distributed throughout the different regions in Belgium. Since one Walloon laboratory did not report the residence of its patients, the representation of the patients group from the Walloon Region is slightly underestimated. However, the heterogeneous distribution should still be taken into consideration in the generalisation of the results to the diagnosed patients in Belgium.

The distribution curve of the number of infected cases as a function of time had the same shape as the one observed by other implemented surveillance systems such as the surveillance SGP network $(3,4)$ and the paediatric surveillance $(5)$. The peak of the number of positive cases was observed in week 43 , after which, in week 44 , the decline of the number of cases was initiated. 
There is some evidence that the start of school holidays reduces the influenza transmission and that the return to school slightly accelerates the epidemic. As the decline in prevalence occurred before the autumn holidays (week 45) we could not confirm this hypothesis.

The similarity in the age distribution of patients at the different laboratories demonstrates the age-independent inclusion criteria for these laboratories (Figure 2).

The vulnerability for Influenza A(H1N1)2009 of the younger age groups as shown in this report, was also observed by the national surveillance SGP network (3) and is consistent with other investigations $(6,7)$. The very low presence of people over 65 years of age is consistent with other investigations (Health Protection Agency, Centers for Disease Control and Prevention). Data suggest that the elderly may to some extent be protected from infection $(8,9,10)$.

As in many other countries, the Influenza $A(H 1 N 1) 2009$ virus was the predominant circulating influenza virus $(6,7,11)$. However 8 Influenza $B$ strains were detected by 2 out of 7 laboratories performing this typing assay. Although these 2 laboratories reported the majority of samples $(53.7 \%)$, this prevalence of subtype B could still be an underestimation. This is of notice as no Influenza B strain was detected from the samples provided by the SGP network (4). Whether this difference was due to the selection of patient samples or to the sensitivity or specificity of the assays should still be investigated.

These data are an additional tool for the virological surveillance of Influenza $A(H 1 N 1) 2009$ during the epidemic in Belgium. While the epidemiological surveillance by the SGP network selects patients with influenza-like illness (ILI) attending the general practitioner's, this surveillance study is focussed on hospitalised patients and patients at risk of severe diseases. Therefore, the obtained laboratory results provide information on the identification of the infection waves during an epidemic and can be implemented as a confirmation tool of the population-based surveillance.

To avoid inappropriate assignment of morbidity to the Influenza $A(H 1 N 1) 2009$ virus, some efforts to detect other respiratory viral agents are warranted. Therefore, it would be of particular interest if this virological surveillance could be enlarged with the monitoring of cocirculating viral agents associated with ILI during the Influenza season. Similarly the link between the cases and the clinical data is lacking in this study and should be considered in a future study.

Quality assessments have proven their usefulness to demonstrate similar performances between the different laboratories $(12,13)$. Considering the heterogeneous PCR procedures used by the different laboratories, the organisation of such a quality assessment will be of upmost importance to compare the relatedness of the performances between the laboratories. Meanwhile such a quality assessment was organised after the epidemic period by the MolecularDiagnostics.be workgroup for their members including most of the participating laboratories from our study. Results are available for these members through their website (14). 
This study demonstrated the fluent decentralisation of the confirmatory analysis from the NIC towards the participating laboratories. The network and websites were successfully organised and implemented allowing the collection of virological data. This network allowed the reduction of the workload at the NIC as well and made the diagnosis more widely available. Despite the differences in testing algorithm and inclusion criteria, similar positivity rates as well as age distributions of the confirmed cases were notified between the laboratories.

\section{Acknowledgement}

We would like to acknowledge following members of the participating laboratories: Farhoudi Moghaddam A., Walter H., and Buysschaert C. (UZ Gent, Gent), Boone E. (Heilig Hartziekenhuis, Roeselare), Schroder R (Cliniques du Sud-Luxembourg, Arlon), Borremans A. and Beuselinck K. (UZ Leuven, Leuven), Antoine M. (Institut de Pathologie et de Génétique, Loverval), Meex C. (CHU Liège, Liège), Soetens O., and Stevens D. (UZ Brussel, Brussels), Raymaekers M. (Jessa Ziekenhuis, campus Virga Jesse, Hasselt.), Duys M., and De Foor M. (CHU St Pierre - J. Bordet Institute, Brussels), Benoy I. and Depuydt Chr. (AML, Antwerpen.), Mertens A. and Bruynseels P. (ZNA Middelheim, Antwerpen), Bogaerts P. and Bouchahrouf W. (Clinique Universitaire UCL de Mont-Godine, Yvoir).

\section{References}

1. Nicoll A, Coulombier D. Europe's initial experience with pandemic (H1N1)2009 mitigation and delaying policies and practices. Euro Surveill. 2009 Jul 23;14(29)

2. Litzroth A, Gutiérrez I, Hammadi S. Influenza A(H1N1)2009 pandemic: Chronology of the events in Belgium. Arch Publ Health 2010; 68(2): 48-52

3. Van Casteren V, Mertens K, Antoine J, Wanyama S, Thomas I, Bossuyt N. Clinical influenza surveillance of Influenza $\mathrm{A}(\mathrm{H} 1 \mathrm{~N} 1) 2009$ pandemic through the network of Sentinel General Practitioners. Arch Publ Health 2010; 68(2): 62-7

4. Gérard C, Brochier B, Quoilin S, Wuillaume Françoise, Van Casteren V, Thomas I. Virological surveillance of Influenza $\mathrm{A}(\mathrm{H} 1 \mathrm{~N} 1) 2009$ pandemic: the roles of the Belgian National Influenza Centre. Arch Public Health 2010; 68(2): 68-75

5. Sabbe M, Hue D, Antoine J, Dupont Y, Van Eldere J, Van Ranst M, Thomas I. Influenza surveillance in children: first experiences with the Belgian Paediatric Surveillance system "PediSurv". Arch Publ Health 2010; 68(3) [in press]

6. Gilsdorf A, Poggensee G; Working Group Pandemic Influenza A(H1N1)v. InfluenzaA(H1N1)v in Germany: the first 10,000 cases. Euro Surveill. 2009 Aug 27;14(34).pii: 19318. PubMed PMID: 19712649

7. Rizzo C, Declich S, Bella A, Caporali MG, Lana S, Pompa MG, Vellucci L, Salmaso S. Enhanced epidemiological surveillance of influenza $\mathrm{A}(\mathrm{H} 1 \mathrm{~N} 1) \mathrm{v}$ in Italy. Euro Surveill 2009 Jul 9;14(27). pii: 19266. PubMed PMID: 19589331

8. Vaillant L, La Ruche G, Tarantola A, Barboza P; epidemic intelligence team at InVS. Epidemiology of fatal cases associated with pandemic H1N1 influenza 2009. Euro Surveill 2009 Aug 20;14(33)

9. Miller MA, Viboud C, Balinska M, Simonsen L. The signature features of influenza pandemics-implications for policy. N Engl J Med 2009 Jun 18;360(25):2595-8. Epub 2009 May 7

10. Pérez-Trallero E, Piñeiro L, Vicente D, Montes M, Cilla G. Residual immunity in older people against the

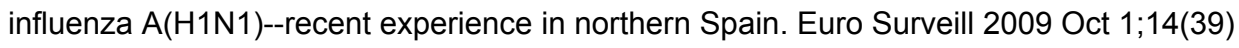


11. Ciblak MA, Albayrak N, Odabas Y, Basak Altas A, Kanturvardar M, Hasoksuz M,Sucakli B, Korukluoglu G, Bal E, Ertek M, Badur S. Cases of influenza A(H1N1)v reported in Turkey, May-July 2009. Euro Surveill. 2009 Aug 13;14(32). pii: 19304. Erratum in: Euro Surveill 2009;14(33)

12. Muyldermans $G$, Soetens $O$, Antoine M, Bruisten S, Vincart B, Doucet-Populaire F, Fry NK, Olcén $P$, Scheftel JM, Senterre JM, van der Zee A, Riffelmann M, Piérard D, Lauwers S. External quality assessment for molecular detection of Bordetella pertussis in European laboratories. J Clin Microbiol 2005 Jan;43(1):30-5

13. Muyldermans G, Debaisieux L, Fransen K, Marissens D, Miller K, Vaira D, Vandamme AM, Vandenbroucke AT, Verhofstede C, Schuurman R, Zissis G, Lauwers S. Blinded, multicenter quality control study for the quantification of human immunodeficiency virus type 1 RNA in plasma by the Belgian AIDS reference laboratories. Clin Microbiol Infect 2000 Apr;6(4):213-7

14. http://www.moleculardiagnostics.be/ 